\title{
Text Region Extraction from Quality Degraded Document Images
}

\author{
S. Abirami and D. Manjula \\ Department of Computer Science \& Engg, Anna University \\ Chennai, India. \\ \{abirami@cs.annauniv.edu, manju@annauniv.edu\}
}

\begin{abstract}
In this paper we present a well designed method that makes use of edge information to extract textual blocks from gray scale document images. It aims at detecting textual regions on heavy noise infected newspaper images and separate them from graphical regions. The algorithm traces the feature points in different entities and then groups those edge points of textual regions. Finally feature based connected component merging was introduced to gather homogeneous textual regions together within the scope of its bounding rectangles. The proposed method can be used to locate text in-group of newspaper images with multiple page layouts. Initial results are encouraging, then they are experimented with considerable number of newspaper images with different layout structures and promising results were obtained. This finds its major application in digital libraries for OCR where information can be of different quality depending on the age of the scanned paper.
\end{abstract}

Keywords: Text Extraction, Edge Detection, Block Merging.

\section{Introduction}

In spite of the wide spread use of computers and other digital facilities, paper document keeps occupying a central place in our everyday life. Segmenting an image into coherent regions is the first step before applying an object recognition method. For example, prior to using an optical character recognizer (OCR), the characters must be extracted from the image. In this paper, the problem of finding text region in a quality degraded newspaper image is discussed. The stated work may be a part of work that facilitates the news article retrieval system.

Newspaper images contain quite a lot of noise, may be one of them was photocopied from the earliest issue of local newspaper dated back or it might have been crushed for some reasons. Isolation of text and graphics is more difficult to do by a segmentation process of a general method. In this paper, we are interested in the preliminary step of document analysis and recognition. Our present goal is to extract textual areas from noisy document images.

We briefly present previous research in the physical segmentation of a document and text detection (in Section 2). Then we describe our work (in Section 3), which mainly consists of detection of zones of interest and discrimination of text areas. Results have been discussed in Section 4. Section 5 shows the performance measures. Section 6 concludes the work. 


\section{Related Works}

Techniques for document segmentation and layout analysis are traditionally subdivided into three main categories: bottom-up, top-down and hybrid techniques. Some other up-to-date methods are introduced by recent progresses in this area, so as to expand the scope of above categorization [4]. Bottom-up techniques [5,6,7] progressively merge evidence at increasing scales to form, e.g., words from characters, lines from words, columns from text lines. Top-down techniques $[8,9,10]$ start by detecting the large-scale features of the image (e.g., columns) and proceed by successive splitting until they reach the smallest-scale features (i.e., individual characters, or text lines).

Most methods do not fit into one of these two categories and are therefore called hybrid. Among these we can find methods based on texture analysis and methods based on background analysis [11].

Text / Graphics separation aims at segmenting the document into two layers: a layer assumed to contain text and a layer containing graphical objects. In [1], a consolidated method proposed by Fletcher and Kasturi, with a number of improvements, to make it more suitable for graphics-rich documents is proposed. As a result of these, we want to extract the textual regions successfully from quality degraded document images, which could be useful for text retrieval systems.

\section{Text Region Extraction}

In this paper, we attempted to extract the text region from quality degraded document images. A high level design of the text region extraction system attempted in this work is shown in figure1. The input to the system could be a color or a gray scale image obtained by scanning the newspapers. Documents containing text, graphics, figures, maps and tables are taken as input. Then the scanned image passes through the following phases.

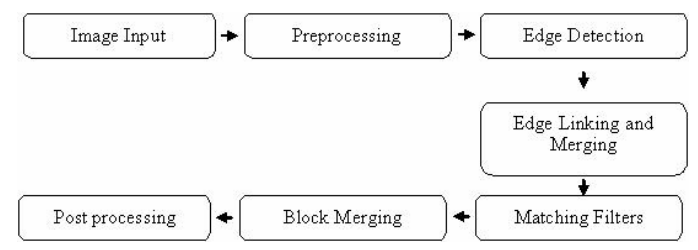

Fig. 1. Text Region Extraction System

\subsection{Preprocessing}

As a first step, input image undergoes preprocessing. If it is a color image, it has to be converted to a grayscale image. Since newspaper images contain noise, it has to be smoothed out. These two steps of grayscale conversion and smoothing, does forms the basic operation in preprocessing. 


\subsubsection{Smoothing}

This step is used to filter out the noises in the original image before the location and detection of edges. In this system, Gaussian filter has been used for smoothing process. The larger the width of the Gaussian mask, the lower is the detector's sensitivity to noise.

\subsection{Edge Detection}

Once the preprocessing gets over, the next step is to detect the edges in the image. In this system, Canny Edge Detector Algorithm is applied for edge detection. The various steps involved in this phase is summarized below.

Edge detection is outlined as:

- $\quad$ Storage of smoothed image. S[i,j]

- Computation of the gradient of smoothed array using $2 * 2$ first difference approximation.

$$
\begin{aligned}
& \text { - } P[i, j]=(S[i, j+1]-S[i, j]+S[i+1, j+1]-S[i+1, j]) / 2 \\
& \text { - } Q[i, j]=(S[i, j]-S[i+1, j]+S[i, j+1]-S[i+1, j+1]) / 2
\end{aligned}
$$

- Calculation of magnitude and orientation for each pixel.

- $\quad M[i, j]=\operatorname{SQRT}(P[i, j] 2+Q[i, j] 2)$

- $\mathrm{O}[\mathrm{i}, \mathrm{j}]=\arctan (\mathrm{Q}[\mathrm{i}, \mathrm{j}], \mathrm{P}[\mathrm{i}, \mathrm{j}])$

- Apply non-maxima suppression to the gradient magnitude.

- $\quad N[i, j]=\operatorname{nms}(M[i, j], L[i, j])$

- Apply suitable threshold to detect and enhance edge.

- $\mathrm{T} 1$ (if needed T2)

To reduce the number of false edge fragments in the non-maxima suppressed gradient magnitude, a threshold has to be applied. All values below the threshold are changed to zero.

\subsection{Edge Linking and Merging}

Once the edge points are detected, with these edge points, links between these edges has to be identified. This process is called edge linking. Once links are determined, small edge lists need to be merged into longer lines called as edge merging. To perform edge linking, connected components of the edge pixels have to be detected. This is done by applying 8-Adjacnecy Connectivity algorithms.

Once the edge links are determined, calculate the difference between a pair of edge links. If the difference remains to be minimum, then merge it to form a single larger path. Finally, for each path, finds its maximum and minimum point and bound it with a rectangle.

\subsection{Matching Filters}

Once Edge linking and merging gets over, filter has been applied to remove lines that don't help in finding text components, edges of the bounding box of graphics or text. 
First, Pixel Chain code has been computed to match the patterns that could form lines to be removed. Next to that, white pixel gradient average for each component has been computed to remove the things that have very small average (Box Filter).

\subsubsection{Pixel Chain Coding}

Features of interest range of feature dimensions, noise dimensions, from the set are selected. This is done with knowledge of the characteristics of desired signal or undesired noise. Short isolated lines and spurs that match the pattern are eliminated. In addition to the advantage of feature-based and contextual filtering, pixel chain coding also provides a computational advantage over pixel-based processing because only one directional information is stored than the two coordinate information for each pixel. Figure 2 shows the directional numbers for a pixel $\mathrm{P}$.

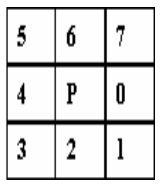

Fig. 2. Pixel Chain Coding

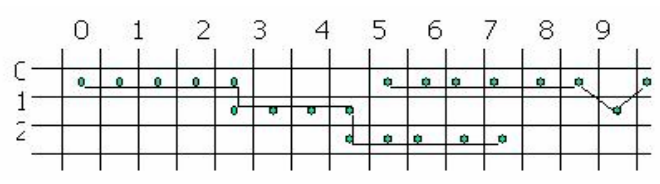

Fig. 3. Matching Filters

Thus the detected links and their corresponding PCC in the above valid edges in figure 3 are as follows

$$
\begin{aligned}
& \text { Link1:- }(0,0)->(7,2) \text { PCC: }-0->0->0->0->0->2->0->0->0->2->0->0->0->0->0 \\
& \text { Link2:- }(5,0)->9,0) \text { PCC: }-0->0->0->0->0->0->1->0
\end{aligned}
$$

With these Chain codes, line patterns are recognized. If the code follows a similar direction value throughout its course, then its pattern does forms a line, which can be deleted. Similarly, if codes with minimal variance match the pattern, they can be deleted making an assumption that the minimal variance could have been caused due to noise. This process is called as line filtering.

\subsubsection{Box Filters}

As the next step the image is subjected to box filtering. In this step, the white pixel average for each component is calculated. If the average number of white pixels available in a component is less than the threshold average being supplied, then the component is considered to represent a graphic, and it is deleted.

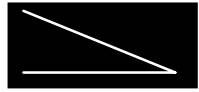

Fig. 4.1. Graphic

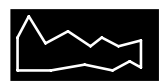

Fig. 4.2. Text

\subsection{Block Merging}

At this stage it is considered that all the components so far detected as text. But it is not certain. Instead of analyzing smaller components, they are merged to form bigger 
blocks by connected component Analysis. Different components of a single block, either graphic or text, closed to each other are connected to form bigger blocks for further analysis.

\subsection{Post Processing}

In this phase, as a post process, histogram analysis has been made on the image to discriminate the graphical regions, further present on the image. Histogram analysis is similar to box filter, with a variation that, the image being considered is a grayscale image. Average number of white pixel distribution is calculated. Since the range of values is more, we calculated the edge gradient average (considering all the gradients) and normalized number of edge gradient pixel in the component more than the threshold. It is determined that, both the measures remain to be higher than the threshold for text components. These measures are calculated as follows. The first feature, edge gradient average, is defined as:

$$
F_{a}=\frac{\sum_{i=T_{a}}^{G_{m}} H(i) \bullet i}{\sum_{i=T_{A}}^{G_{m}} H(i)}
$$

Where Th is the threshold value, Gm is the maximum value of the gradient, and $\mathrm{H}(\mathrm{i})$ is the histogram of the edge gradient. The second feature Fn, normalized number of edge pixels, is defined as:

$$
F_{n}=\frac{\sum_{i=T_{n}}^{G_{m}} H(i)}{N_{x} \bullet N_{y}}
$$

Where $N x, N y$ are the width and the height of the block, respectively. This feature represents the density of the edge pixels in a block. In general, the graphic blocks, which contain much blank space, has the lower density of edge pixels in contrast to the text blocks. So, we can use $F n$ to discriminate graphic block from the text block. Histogram of each component is drawn and analyzed for regularity of extremes. If the regularity is smooth with minimal variance, then it is concluded as text and for irregular extremes, it is considered as graphics and eliminated. Those components that remain form the output. An example is given in the figures 5.1 and 5.2.

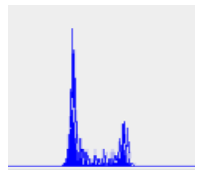

Fig. 5.1. Graphic Block

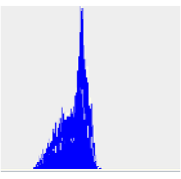

Fig. 5.2. Text Block

\section{Experiments and Results}

This system has been implemented using Java Advanced Imaging. A set of 1000 images from different newspapers have been scanned at a resolution less than 200 


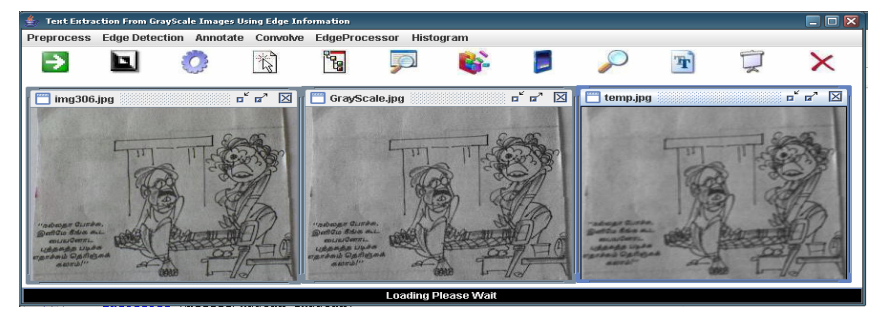

Fig. 6. Preprocessed Image

dots per inch have been tested in this system and promising results were obtained. When an input image is supplied to the system, it undergoes preprocessing, which is shown in figure 6.

Canny edge detection algorithm is applied on the image to enhance the valid edge points. Valid edges are subjected to connectivity algorithm, to find the edge links. After merging, links lying in close proximity with minimal discontinuation are made as a bigger path and bounded by rectangular box. Figure 7 shows the image after edge linking and merging. Connected Component Analysis is performed with the neighboring blocks in horizontal direction and merged together to form a bigger component. Figure 8 shows the image after block merging process.

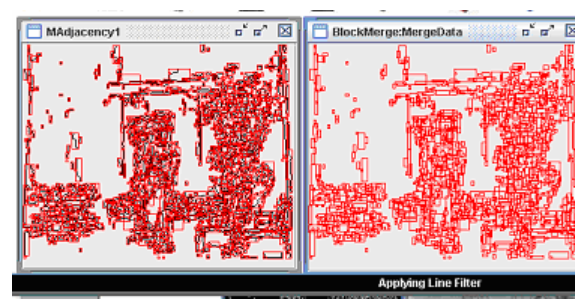

Fig. 7. Edge Linking and Merging

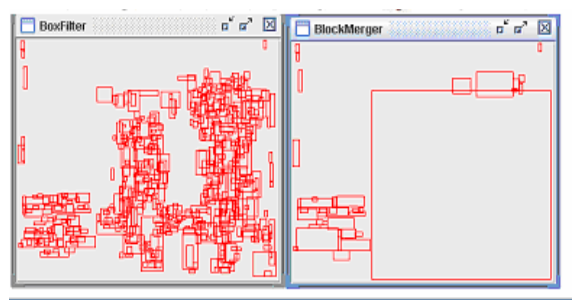

Fig. 8. Block Merging

As a final step, the bigger components are subjected to a histogram processing where graphical components are discriminated and removed. Extracted Text region is shown in Figure 9.

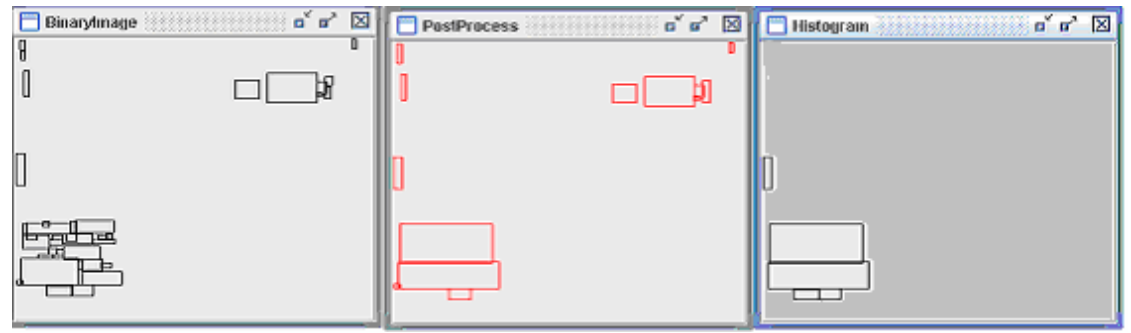

Fig. 9. Extracted Text Region 


\section{Performance Measurements}

A set of 1000 images from different newspapers have been scanned at a resolution less than 200 dots per inch have been tested in this system and promising results were obtained. Test Data and Results of some of the images have been projected in the table 1 .

Table 1. Test Data and Results

\begin{tabular}{|c|c|c|c|c|c|c|c|c|c|c|c|}
\hline $\begin{array}{l}\text { Image } \\
\text { Name }\end{array}$ & $\begin{array}{c}\text { Gau } \\
\text { ssian }\end{array}$ & $\mathbf{F}$ & $\begin{array}{l}\text { PBF } \\
T\end{array}$ & $\begin{array}{l}\text { SBF } \\
\mathbf{T}\end{array}$ & $\begin{array}{r}\mathbf{P P} \\
(\mathbf{X x Y}) \\
\end{array}$ & HT & TAR & TACR & $\begin{array}{r}\text { TA } \\
\text { WR }\end{array}$ & \begin{tabular}{|r|}
$\mathbf{T}$ \\
TAN \\
$\mathbf{R}$ \\
\end{tabular} & $\begin{array}{r}\mathbf{T T} \\
\mathbf{A R} \\
\end{array}$ \\
\hline img 18 & $\mathrm{Y}$ & $\mathrm{N}$ & 0.09 & 0.13 & $3 \times 3$ & 0.5 & 955 & 955 & 0 & 0 & 955 \\
\hline img 122 & $\mathrm{Y}$ & $\mathrm{Y}$ & 0.1 & 0.12 & $3 \times 3$ & 0.3 & 601 & 601 & 0 & 20 & 621 \\
\hline $\operatorname{img} 233$ & $\mathrm{Y}$ & $\mathrm{YN}$ & & 0.13 & $4 \times 4$ & 0.3 & 3437 & 3426 & 11 & 30 & $\begin{array}{r}346 \\
7 \\
\end{array}$ \\
\hline img 419 & $\mathrm{Y}$ & $\mathrm{Y}$ & 0.09 & 0.1 & $3 \times 3$ & 0.5 & 1663 & 1602 & 61 & 45 & $\begin{array}{r}170 \\
8 \\
\end{array}$ \\
\hline $\operatorname{img} 512$ & $\mathrm{Y}$ & $\mathrm{Y}$ & 0.14 & 0.11 & $2 \times 2$ & 0.4 & 1973 & 1960 & 13 & 40 & $\begin{array}{r}201 \\
0 \\
\end{array}$ \\
\hline img 640 & $\mathrm{Y}$ & $\mathrm{Y}$ & 0.1 & 0.11 & $4 \times 4$ & 0.55 & 678 & 678 & 0 & 30 & 708 \\
\hline img 67 & $\mathrm{Y}$ & $\mathrm{Y}$ & 0.09 & 0.11 & $5 \times 5$ & 0.3 & 1666 & 1666 & 0 & 60 & $\begin{array}{r}172 \\
6 \\
\end{array}$ \\
\hline img 188 & $\mathrm{Y}$ & $\mathrm{Y}$ & 0.09 & 0.11 & $4 \times 4$ & 0.15 & 1024 & 1024 & 0 & 45 & $\begin{array}{r}106 \\
9 \\
\end{array}$ \\
\hline img 99 & $\mathrm{Y}$ & $\mathrm{NN}$ & & 0.1 & $3 \times 3$ & 0.2 & 1109 & 1109 & 0 & 30 & $\begin{array}{r}113 \\
9 \\
\end{array}$ \\
\hline img 300 & $\mathrm{Y}$ & $\mathrm{Y}$ & 0.13 & 0.13 & $4 \times 4$ & 0.26 & 753 & 463 & 290 & 20 & 773 \\
\hline $\mathbf{L F}$ & \multicolumn{5}{|c|}{ Line Filter } & $\begin{array}{r}\text { TTAF } \\
\text { Retrieved }\end{array}$ & 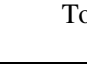 & al Text & Area $\mathrm{T}$ & To be & \\
\hline PBFT & \multicolumn{5}{|c|}{ Primary Box Filter Threshold } & \multicolumn{5}{|c|}{ Precision $=($ TACR/TAR $) * 100$} & \\
\hline SBFT & \multicolumn{5}{|c|}{ Secondary Box Filter Threshold } & \multicolumn{5}{|c|}{ Recall $=($ TACR/TTAR $) * 100$} & \\
\hline $\begin{array}{l}\mathbf{P P}(\mathrm{X} x \\
\end{array}$ & \multicolumn{5}{|c|}{ Post Process $(\mathrm{X}$ x Y) } & \multicolumn{5}{|c|}{ Total Area Correctly Retrieved } & \\
\hline HT & \multicolumn{5}{|c|}{ Histogram Threshold } & \multicolumn{5}{|c|}{ TAWR Total Area Wrongly Retrieved } & \\
\hline TAR & \multicolumn{5}{|c|}{ Total Area Retrieved } & $\begin{array}{r}\text { TTAI } \\
\text { Retrieved }\end{array}$ & R Tot: & Text & C27Area & Not & \\
\hline
\end{tabular}

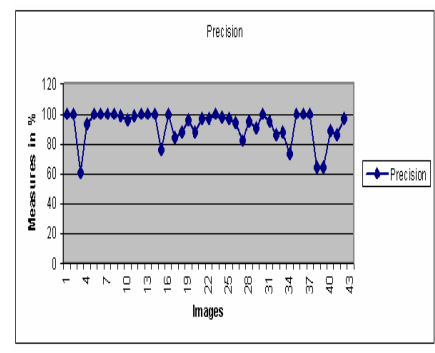

Fig. 10. Precision Graph

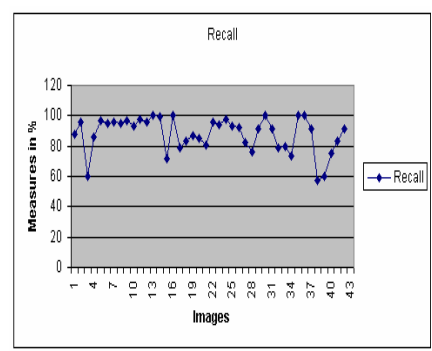

Fig. 11. Recall Graph 
Figure $10 \& 11$ represents the precision and recall graph, which depicts the text area extracted by the system in percentage.

The precision and recall of those corresponding images tested as per table 1 are given in the following table 2 in measures of percentage. Figure 12 represents various precision and recall measures obtained for various test images with reference to the table 2.

Table 2. Precision Vs. Recall

\begin{tabular}{|c|c|c|}
\hline $\begin{array}{l}\text { Image } \\
\text { Pame }\end{array}$ & $\begin{array}{l}\text { Recall } \\
\text { in } 90\end{array}$ & $\begin{array}{l}\text { Precision } \\
\text { in } 96\end{array}$ \\
\hline $\begin{array}{c}\text { irreg } 18 \\
\text { irng } 122\end{array}$ & $\begin{array}{l}88 \\
96\end{array}$ & $\begin{array}{l}100 \\
1000\end{array}$ \\
\hline irreg 23 & $\begin{array}{l}97 \\
97\end{array}$ & 10 \\
\hline irrs: 512 & 93 & 96 \\
\hline $\begin{array}{l}\text { irmeg } 640 \\
\text { irng } 67\end{array}$ & $\begin{array}{l}98 \\
81\end{array}$ & $\begin{array}{l}99 \\
97\end{array}$ \\
\hline $\begin{array}{c}\text { irreg } 188 \\
\text { irng } 99\end{array}$ & $\begin{array}{l}96 \\
94\end{array}$ & $\begin{array}{l}97 \\
1010\end{array}$ \\
\hline irng 300 & 98 & 98 \\
\hline
\end{tabular}

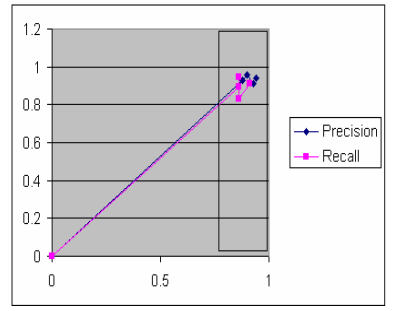

Fig. 12. Precision Vs Recall

\section{Conclusion and Future Work}

Current OCR techniques and other document segmentation and recognition techniques do not work well for documents with text printed under shaded or textured backgrounds or those with non structural layouts. We proposed a Text region extraction, which works well for the documents even with degraded quality.

The main benefits of our method fall on its efficiency for less memory usage and fast running speed. Through this, text retrieval system can have an efficient access over the text. One of major weakness of our algorithm resides on its assumption that all text is oriented in the same direction, which is by default horizontal. From experiments we found that the ratio of successful detection drops down gradually in exceptional cases when textual paragraphs intertwined heavily with irregular graphical blocks. As a future enhancement, instead of applying global threshold as followed in this system, adaptive thresholds can be used to get further refinement. If these are done successfully, we shall hope this system could play a vital role in newspaper retrieval system and digital library system invariable of the quality of the documents being maintained from past. 


\section{References}

1. Tombre, K., Tabbone, S., Lamiroy, B.: Text / Graphics Separation Revisited (2002)

2. Chanda, S., Pal, U.: English, Devnagari and Urdu Text Identification (August 2003)

3. Kasthuri, R., O' Gorman, L., Govindaraju, V.: Document Image Analysis - A Primer

4. Chan, C.H., Pau, L.F., Wang, P.S.P.: Handbook of Pattern Recognition \& Computer Vision, 2nd edn. (1999)

5. Pavlidis, T., Zhou, J.: Page Segmentation and Classification. Computer Vision Graphics Image Processing 54(6), 484-496 (1992)

6. Wang, D., Srihari, S.N.: Classification of newspaper image blocks using texture analysis. Computer Vision, Graphics, and Image Processing 47, 327-352 (1989)

7. Fletcher, L.A., Kasturi, R.: A robust algorithm for text string separation from mixed text/graphics images. IEEE Transaction on Pattern Analysis and Machine Intelligence 10(6), 910-918 (1988)

8. Le, D.X., Thoma, G.R.: Document classification using connectionist models. In: Proc. of IEEE International Conference on Neural Networks, Orlando, Florida, vol. 5, pp. 3009 3014 (June 1994)

9. Ohya, J., Shio, A., Akamatsu, S.: Recognizing Characters in Scene Images. IEEE Transaction on PAMI 16(2), 214-224 (1994)

10. Baird, W.S., Jones, S.E., Fortune, S.J.: Image segmentation by shape directed covers. In: Proc. Of ICPR, pp. 820-825 (1990)

11. Jain, K., Bhattacharjee, S.: Text Segmentation Using Gabor Filters for Automatic Document Processing. Machine Vision and Applications 5(3), 169-184 (1992) 